\title{
The Repulsive Dark Matter Model of the Universe Relates the Hubble Constant to the Temperature of the CMBR
}

\author{
Jaroslav Hynecek ${ }^{1}$ \\ ${ }^{1}$ Isetex, Inc., 905 Pampa Drive, Allen, USA \\ Correspondence: Jaroslav Hynecek, Isetex, Inc., 905 Pampa Drive, Allen, TX 75013, USA. E-mail: \\ jhynecek@netscape.net
}

Received: October 7, 2012 Accepted: October 25, 2012 Online Published: March 21, 2013

doi:10.5539/apr.v5n2p76 URL: http://dx.doi.org/10.5539/apr.v5n2p76

\begin{abstract}
This paper describes the derivation of the fundamental quantum of Repulsive Dark Matter, its relation to the Hubble constant, and to the Cosmic Microwave Background Radiation (CMBR). It is shown, based on simple principles, that the Hubble constant is not an independent cosmological parameter but that it is intimately related to the temperature of the CMBR. This finding thus allows to precisely calculate the Hubble constant from the temperature that is accurately measured and that the current Big Bang theory of the universe cannot offer.
\end{abstract}

Keywords: cosmic microwave background radiation, Hubble constant, repulsive dark matter, Hubble mass of the universe, Hubble distance, fundamental quantum of dark matter, Big Bang theory, neutrino mass

\section{Introduction}

In the previously published papers by Hynecek $(2009,2012)$ it was shown that it is possible to develop a model of the universe that is based on the idea of finite non-expanding sphere of repulsive dark (dark = transparent) matter (RDM), in which the galaxies move from the bulk to the surface. It was shown that such a model agrees well with many observations, for example, the observations of Gamma Ray Bursts (GRB). To avoid confusion with the standard DM that is postulated in the main stream literature it is necessary to emphasize that the depletion of the repulsive DM from its local uniform background that this model is using is equivalent to the action of the standard attractive DM that seems to aggregate in and around the galaxies. Therefore, the depletion of RDM from the uniform background $\approx$ standard attractive DM in the zero background. The repulsive DM model makes many additional comparisons with observations that are not easily explained in the Big Bang theory, such as the duration of the long GRB pulses (Hynecek, 2012), but this will not be discussed here.

One more comment, however, more or less of a philosophical nature on the finite size of the RDM model of the universe is necessary, because this model is such a significant departure from the commonly accepted Big Bang model that is being taught at every university, and because there is a natural tendency of every human being to ask what is beyond. The answer is that we do not know and at this time it is in principle impossible to know, because no data can be retrieved from the "region beyond". The ordinary matter, including the human beings, exist and light propagates only in the space-time where the RDM is present. The notion of "beyond" itself comes only from our experiences with the natural space-time we are living in, and thus only from the RDM space. Therefore, because there is no space-time as we know it beyond the RDM sphere, the question itself "what is beyond" does not make sense and logically cannot be asked because there is no beyond.

Returning now back to the details of the RDM model; both referenced papers derive the relation between the repulsive dark matter density $m_{0}$ and the Hubble constant $H_{0}$, which characterizes the well known dependence of recession velocity of the galaxies on their distance from Earth, as follows:

$$
H_{0}=\sqrt{\frac{8}{3} \pi \kappa m_{0}}
$$

In this formula $\kappa$ is the Newton gravitation constant. From the simple dimensional analysis it is also possible to define other Hubble parameters that can be useful in simplifying various cosmological equations. For example, the physical Hubble distance $\rho_{h}$, where $\rho$ is found from the differential equation: $d \rho=g_{t t}{ }^{-1 / 2} d r$, can be defined as:

$$
\rho_{h}=2 c / H_{0}
$$


with $c$ being the intergalactic speed of light measured in the vicinity of the Milky Way galaxy. Similarly, for the total Hubble RDM mass of the entire universe, that now has a finite size, it was derived that it is equal to:

$$
M_{h}=\frac{4 \pi}{3} \rho_{h}^{3} m_{0}=4 c^{3} / \kappa H_{0}
$$

and finally, for the Hubble time it is customary to write:

$$
\tau_{h}=1 / H_{0}
$$

The Hubble constant can, therefore, be considered also as a nature's ultimate low frequency and used for normalization to form a dimensionless frequency constant of the universe:

$$
N_{\omega}=\frac{k_{B} T_{0}}{g_{t t} h H_{0}}=8.42364 \cdot 10^{29}
$$

In this expression the parameter $h$ is the well known Planck constant and $g_{t t}$ is the metric coefficient standing by the natural time coordinate differential $d t$ in the metric line element: $d s^{2}=g_{t t}(c d t)^{2}-g_{t t}{ }^{-1} d r^{2}-g_{t t} \rho^{2} d \Omega^{2}$ of the universe's spherically symmetrical space-time. The meaning of this frequency constant will be explained in more detail later.

\section{Additional Hubble Parameters}

The definitions of various Hubble parameters do not have to be limited to the definitions of the Hubble time, the Hubble mass of the universe, and the dimensionless frequency constant. The definitions can be extended to other interesting parameters such as the Planck-Hubble DM mass quantum:

$$
m_{q h}=h H_{0} / c^{2}=1.625 \cdot 10^{-68} \mathrm{~kg}
$$

Once this parameter is established it is interesting to investigate its dimensionless ratio to the universe's total Hubble RDM mass defined above in Equation 3:

$$
N_{p h}=\frac{M_{h}}{m_{q h}}=\frac{4 c^{5}}{\kappa h H_{0}^{2}}=4.520 \cdot 10^{121}
$$

If this number is recognized as another fundamental constant of the universe, the nature's largest number, then the Hubble constant could be simply calculated as:

$$
H_{0}=\sqrt{\frac{4 c^{5}}{\kappa h N_{p h}}}
$$

or alternately, the Planck constant would depend on the Hubble constant or on the CMBR temperature $T_{0}$, as will become clear later:

$$
h=\frac{4 c^{5}}{N_{p h} \kappa H_{0}^{2}}=\frac{4 \pi}{3} \frac{\kappa}{c}\left(\frac{k_{B} T_{0}}{c^{2} g_{t t}}\right)^{2} \sqrt{N_{p h} / \pi}
$$

The term in the parenthesis is yet another mass quantum equal to: $k_{B} T_{0} / c^{2} g_{t t}=1.368 \cdot 10^{-38} \mathrm{~kg}$. This mass quantum will be later identified with the fundamental RDM mass quantum $m_{q \lambda}$. The constants $N_{p h}$ and $N_{\omega}$ however, are not independent constants, they are related to each other according to the formula: $N_{p h} / \pi=(256 / 9) N_{\omega}{ }^{4}$.

An obvious question that these expressions suggest is the question of the long time universe behavior. Assuming that the fundamental constants $N_{p h}$ and $N_{\omega}$ are really constant and do not change with the cosmological time, it would be interesting to investigate whether the CMBR temperature on the long range time scale is actually stable, as it should be in any thermodynamically isolated closed system, and only the universe's overall entropy is changing. This investigation will be left for the future work.

It is also interesting to calculate the ratios of $m_{q h}$ to the neutron mass and to the recently published combined upper limit mass $m_{n i}$ of the three neutrino variety estimated to be: $0.28 \mathrm{eV}$. 


$$
m_{n} / m_{q h}=1.031 \cdot 10^{41}, m_{n i} / m_{q h}=3.072 \cdot 10^{31}
$$

\section{The Fundamental Mass Quantum}

However, another, and much more interesting fundamental RDM mass quantum can be found by assuming that the RDM forms almost a mass-less crystal-like structure consisting of cells with the standing wave vibrations. It can be further assumed that the mass of these cells is generated by the energy of the vibrations themselves and that any other mass that might be the part of the cell structure is much smaller and can be neglected. An example of such cell vibrations might be the trapped photons that, as is well known, exert a repulsive pressure on the cell walls. Such a photon pressure would then be consistent with the repulsive force of the RDM on the visible ordinary matter, while the RDM mass would be attracted to itself. Considering next that the cells may have random shapes and sizes, but on the average most likely resemble the diamond or cubic crystals, the equation for the fundamental RDM mass quantum can then be derived in the following way: Defining the volume of the average crystal primitive cell as $V_{0}$, and the wavelength of the cell elements vibrations as $\lambda$, the average RDM mass energy $E_{0}$ contained in the cell is:

$$
E_{0}=m_{0} V_{0} c^{2}=h c / \lambda
$$

The average unit cell volume will now be related to the wavelength by considering that the cell elements have a certain packing density $1 / \xi$, where $\xi$ can also be thought of as a spatial degeneracy. An example of such a degeneracy are the three orthogonal vibrations with the same frequency in a crystal primitive cell. The cell volume is thus simply equal to:

$$
V_{0}=\xi(\lambda / 2)^{3}
$$

From Equation 11 and Equation 12 then follows the formula for the wavelength:

$$
\left(\frac{\lambda}{2}\right)^{4}=\frac{h}{2 m_{0} c \xi}
$$

It is now simple to find equation for the fundamental RDM mass quantum as follows:

$$
m_{q \lambda}=m_{0} \xi\left(\frac{\lambda}{2}\right)^{3}=\sqrt[4]{\frac{\xi m_{0} h^{3}}{8 c^{3}}}
$$

For the diamond cell the packing density is well known and, therefore, for $\xi$ holds that:

$$
\xi=\frac{16}{\pi \sqrt{3}}=2.940421
$$

However, the value $\xi=3$ is probably more likely because it describes the exact threefold spatial degeneracy that corresponds to the three spatial dimension. Knowing now the precise value of the Hubble constant, the RDM mass density and from that the RDM mass quantum can be found. The most probable value for the Hubble constant found in the literature used previously by Hynecek (2009) is: $H_{0}=68.0 \mathrm{~km} / \mathrm{secMpc}$. This value was published by Murphy et al. (2009), and is close to the value of: $H_{0}=67.922 \mathrm{~km} / \mathrm{secMpc}$ derived below in this article from the measured CMBR temperature: $T_{0}=2.7255^{\circ} \mathrm{K}$, published by Fixsen (2009). Using Equation 1 the RDM mass density becomes:

$$
m_{0}=0.866554 \cdot 10^{-26} \mathrm{~kg} / \mathrm{m}^{3}
$$

and from that using Equation 14 the RDM mass quantum that will be called "nevitron" is:

$$
m_{q \lambda}=1.368621 \cdot 10^{-38} \mathrm{~kg}=7.677 \mathrm{meV}
$$

This mass may be close to the mass of one of the neutrinos, because the upper limit for the combined mass of all three neutrino variety, as already mentioned, is: $m_{n i}=280 \mathrm{meV}$.

It is interesting to consider further that there might be an even deeper analogy with the standard crystalline matter. The neutrinos might be the RDM vacancies corresponding to the light and heavy holes similarly as, for 
example, in the germanium or silicon crystals. The detail study and discussion of such possibilities is left for the future work.

\section{Cosmic Microwave Background Radiation Temperature}

In the previously referenced papers by Hynecek $(2009,2012)$ it was also shown that the CMBR is not the remnant of the Big Bang but that it is an image of the relatively thick surface region that envelopes the entire universe. The universe is thus a solid-like RDM sphere with a finite diameter as already mentioned. The galaxies and any visible or ordinary condensed matter move in this sphere as defects do in a crystal, floating in a free fall from the bulk to the edge where they disintegrate. The border region has a deep negative gravitational potential and thus harbors the remnants of stars or remnants of the entire galaxy explosions and perhaps also various elementary particles, for example neutrons, that cannot escape. Only the radiation such as the thermal radiation or the GRB radiation can escape and is detected here on Earth. By extending the previous simple model of the universe with the uniform RDM density developed by Hynecek (2009) to a model where the RDM is deformable (Hynecek, 2012) it was possible to determine the more precise value for the gravitational potential at the universe's edge:

$$
\varphi_{n}=-1.7436 \cdot c^{2}
$$

instead of the previously derived value of: $\varphi_{n}=-2 c^{2}$. This now allows to find the more precise value also for the time metric coefficient $g_{t t}$ at the universe's edge. The metric coefficients depend on the gravitational potential, and because the universe is not flat, particularly at large distances, the time coordinate metric coefficient $g_{t t}$ is equal to:

$$
g_{t t}=e^{2 \varphi_{n} / c^{2}}=0.030586 \ldots
$$

It is now simple to convert the nevitron mass to energy and the energy to an equivalent temperature at the edge of the universe as observed from Earth. The derivation details are shown in the Appendix. The CMBR temperature that should be observed on Earth as calculated from the parameters that were all derived from the Hubble constant: $H_{0}=68.0 \mathrm{~km} / \mathrm{secMpc}$ is then:

$$
T_{C M B R}=\frac{m_{q \lambda} c^{2} g_{t t}}{k_{B}}=2.727107^{\circ} \mathrm{K}
$$

where $k_{B}$ is the well known Boltzmann constant. The temperature agrees well with the measured CMBR temperature found in the literature: $T_{0}=2.7255 \pm 0.0006^{\circ} \mathrm{K}$ as published by Fixsen (2009). This is a stunning result with a good accuracy that clearly provides the solid and incontrovertible observational proof for the existence of the fundamental RDM mass quantum, nevitron, and for the validity of the developed RDM model of the universe.

It is thus apparent that the CMBR thermal oscillations of the ordinary radiating matter that has accumulated in the shell enveloping the entire universe are in a thermal equilibrium with the RDM thermal vibrations throughout the universe's bulk. These vibrations thus give the RDM its gravitating mass, which then determines the value of the Hubble constant. The existence of Hubble constant that follows from the observation of receding galaxies is thus the clear and unavoidable consequence of the finite and thermodynamically isolated universe.

The precise value for the Hubble constant that is currently not that accurately known can then be directly determined from the CMBR temperature according to the following formula:

$$
H_{0}=\frac{8}{3} \sqrt{\frac{\pi \kappa h}{c^{5}}}\left(\frac{k_{B} T_{0}}{h g_{t t}}\right)^{2}=67.922 \mathrm{~km} / \mathrm{sec} M p c
$$

However, a few more iterations in calculations ought be made to obtain the final accurate value, because the metric coefficient $g_{t t}$ also depends on the Hubble constant and therefore also on the CMBR temperature.

The expression: $k_{B} T_{0} / h g_{t t}$ in Equation 21 can be thought of as a resonant frequency: $v=1.856 \mathrm{THz}$ of the average cell, or when compared to Hubble constant as another fundamental constant of the universe as defined previously in Equation 5. It might be worthwhile to search the RF background noise spectrum or the absorption spectrum in a large volume resonator filled by a mass that couples to the RDM for resonances occurring in the vicinity of this frequency. It might also be interesting to generate strong and possibly phase directed vibrations at that frequency and measure the force exerted on such a vibrating matter. An example of the terahertz absorption spectrum of a relatively small volume without any significant mass that would couple to RDM is shown in Figure 1. 
Similarly, the formula for the RDM mass density becomes:

$$
m_{0}=\frac{8}{3} \frac{h}{c^{5}}\left(\frac{k_{B} T_{0}}{h g_{t t}}\right)^{4}=\frac{8}{3} \frac{c^{3}}{h^{3}}\left(\frac{k_{B} T_{0}}{c^{2} g_{t t}}\right)^{4}=0.866554 \cdot 10^{-26} \mathrm{~kg} / \mathrm{m}^{3}
$$

When this expression is substituted into Equation 14, the formula for the mass quantum $m_{q \lambda}$ becomes:

$$
m_{q \lambda}=k_{B} T_{0} / c^{2} g_{t t}=7.677 \mathrm{meV}
$$

The mass density $m_{0}$ was previously calculated directly from the Hubble constant according to Equation 1 . When the diamond packing density is used for the calculation of these values instead of $\xi=3$ the results become: $H_{0}=$ $68.606 \mathrm{~km} / \mathrm{secMpc}$, and: $m_{0}=0.884112 \cdot 10^{-26} \mathrm{~kg} / \mathrm{m}^{3}$. At this time it is still difficult to determine from observations the correct value of $\xi$, however, in either case, the value of $H_{0}$ is definitely slightly smaller than what is claimed in the main stream literature, for example, by Freedman and Madore (2011): $H_{0}=73.0$ $\mathrm{km} / \mathrm{secMpc}$, or in the Big Bang theory: $H_{0}=70.4 \mathrm{~km} / \mathrm{secMpc}$.

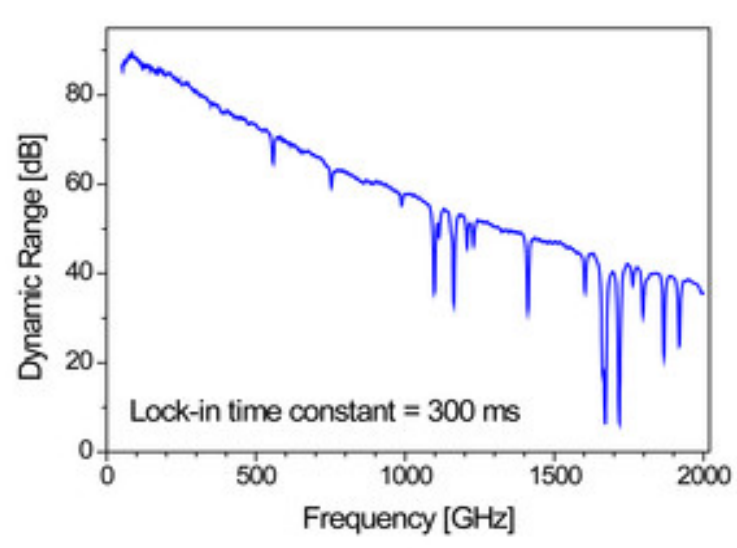

Figure 1. An example of the terahertz frequency absorption spectrum from the spectrometer manufactured by the Toptica Photonics company (2012, web advertisement). The dips in the spectrum are presumably caused by the water vapor, the one before the last is coincidentally at the frequency of $1.86 \mathrm{THz}$. Is it really a coincidence?

\section{Conclusions}

The repulsive DM model of the universe is a finite sphere of a crystalline-like matter enclosed by a shell of radiating material that is in a thermal equilibrium with the RDM throughout the universe via the gravitational coupling. The CMBR originates from this shell rather than being the remnant of the Big Bang. The heat energy of the CMBR gives the RDM its mass. When galaxies collide with this shell the GRBs are generated and are also radiated back to the universe's bulk where they contribute to the generation of new visible or ordinary matter. This model allows to find the value for the fundamental RDM mass quantum called "nevitron" and to find the relation between the Hubble constant and the CMBR temperature. It is not generally believed that the Hubble constant and the CMBR temperature are in any way related to each other. This paper clearly shows that this is not so and that these two parameters are closely related. The determination of one gives automatically the value for the other. Because the CMBR temperature is measured with a very high precision the Hubble constant and the rest of the Hubble parameters are thus also precisely determined. Knowing the precise value of the Hubble constant may eventually lead to the dismissal of the Big Bang theory in favor of the steady state model similar, for example, to the universe described by Hoyle, Burbidge, and Narlikar (2000), as a viable model that correctly describes the reality we are living in. The stunning agreement of observations with this theory and the possibility to calculate the Hubble constant also clearly confirms the original assumption that the universe is a closed system with a finite size. The similar derivation is in principle not possible in the open system that the Big Bang theory represents. There is no similar mass-energy conservation in that theory as it is maintained in the RDM model, which is one of many well known problems of the Big Bang theory. 


\section{References}

Fixsen, D. J. (2009). Retrieved from arXiv:11.1955v2, [astro-ph.CO] 10 Nov 2009.4.

Freedman, W. L., \& Madore, B. F. (2011). The Hubble Constant. Annu. Rev. Astro. Astrophys, 2010(48), 673-710. Retrieved from www.annualreviews.org by California Institute of Technology on 04/03/11

Hoyle, F. G., Burbidge, F. G., \& Narlikar, J. V. (2000). A Different Approach to Cosmology. Cambridge, U.K.: Cambridge University Press.

Hynecek, J. (2009). Uniform dark matter density model of the Universe. Physics Essays, 22(3), 325. http://dx.doi.org/10.4006/1.3157087

Hynecek, J. (2012). Repulsive dark matter model of the universe. Physics Essays, 25(4), 561. http://dx.doi.org/10.4006/0836-1398-25.4.561

Murphy, M. T., Webb, J. K., Flambaum, V. V., Dzuba, V. A., Churchill, C. W., Prochaska, J. X., ... Wolfe, A. M. (2001). Possible evidence for a variable fine structure constant from QSO absorption lines: motivations, analysis and results. Retrieved 4 Sept, 2001 from arXiv:astro=ph/0012419v5

Okun', L. B., Selivanov, K. G., \& Telegdi, V. L. (1999). PHYS-USP, 42(10), 1045-1050. Retrieved from http://www.toptica.com/products/terahertz_generation/lasers_and_photomixers_for_cw_terahertzgeneration /cw_terahertz_spectroscopy_kit.html

\section{Appendix}

In the derivation of the formula in Equation 20, for the conversion of fundamental RDM mass quantum to the equivalent temperature of radiation that is observed on Earth, it is necessary to use two kinds of parameters. The first kind are the parameters defined on Earth and the second kind are the parameters at the universe's edge. These parameters have the index " $i$ " and can be determined when the metric coefficient $g_{t t}$ is known.

For the photon energy $E$ and the momentum $p$ at the universe's edge it holds that:

$$
\begin{gathered}
E_{i}=E \sqrt{g_{t t}} \\
p_{i}=\frac{h_{i}}{\lambda_{i}}=\frac{h}{\lambda \sqrt{g_{t t}}}=\frac{p}{\sqrt{g_{t t}}}
\end{gathered}
$$

The Planck constant is an universal constant having the same value everywhere in the universe as is well known and as can be also found in publications by Hynecek (2001) and Murphy (2001). For the speed of light at the universe's edge, as referenced to the Earth centered coordinate system, it holds that:

$$
d r_{i} / d t_{i}=c_{i}=c g_{t t}
$$

This relation is derived by assuming that Earth and the Milky Way galaxy are located relatively near the center of the universe and that the gravitational corrections due to the Earth's gravity, the Sun's gravity, or any other gravitational potential effect of the Milky Way galaxy stars can be neglected. Equation A3 then follows directly from the general form of metric for the spherically symmetric space-time in a radial direction when the metric line element is set to zero: $d s=0$.

$$
0=d s^{2}=g_{t t}\left(c d t_{i}\right)^{2}-g_{t t}^{-1}\left(d r_{i}\right)^{2}
$$

For the local observer, however, his speed of light $c_{l o}$ is derived from the equation:

$$
c=\frac{d s}{d \tau}=\frac{d s}{d t_{l o}} \frac{d t_{l o}}{d \tau}=\frac{c_{l o}}{\sqrt{g_{t t}}}
$$


Equations A1 and A2 must be consistent with Equation A3 and the photon momentum definition formulas. For more clarity the values of various parameters at the edge of the universe as they relate to values observed by distant or local observers are shown in Table T1. The transformation formulas must be, of course, consistent with the following equations for photons: $\lambda v=c, \Delta p \Delta \lambda=h / 4 \pi, \Delta E \Delta t=h / 4 \pi, p=E / c, E=k_{B} T, E=h v, c^{2}=1 / \varepsilon \mu$, and keep them form-invariant. For the mass quantum at the universe's edge a similar formula as in Equation A2 holds true, as was derived previously by Hynecek (2001) for any stationary inertial mass:

$$
m_{i}=m_{q \lambda} / \sqrt{g_{t t}}
$$

The photon energy that is in a thermal equilibrium with the shell's vibration energy: $E_{i}=m_{i} c_{i}^{2}$ at the universe's edge and is measured on Earth is then determined from the following equation:

$$
E=\frac{E_{i}}{\sqrt{g_{t t}}}=\frac{m_{q \lambda} c^{2} g_{t t}^{2}}{\sqrt{g_{t t}} \sqrt{g_{t t}}}=m_{q \lambda} c^{2} g_{t t}
$$

This energy is equivalent to a temperature resulting in the formula used in Equation 20:

$$
T_{C M B R}=\frac{m_{q \lambda} c^{2} g_{t t}}{k_{B}}
$$

where $k_{B}$ is the Earth referenced Boltzmann constant. It is worth mentioning, however, that the Boltzmann constant similarly as the Planck constant and the fine structure constant is a universal constant having the same value anywhere in the universe, Murphy at al. (2001). It is therefore not important to which coordinate system it is referenced to.

In conclusion of this section it is necessary to emphasize that the RDM can interact only with the visible matter, any other ordinary condensed matter, or with another RDM, and does not directly interact with photons by exchanging any energy with them even though the photons propagate in this matter as the transversal waves. The RDM also does not interact with any other "energy" or quasi-particles that move at the speed of light and, therefore, do not poses the gravitational mass as explained by Okun', Selivanov, and Telegdi (1999). This also includes, for example, the hypothetical gravitons. But the RDM is attractive to itself and curves the natural space-time through its gravitational action. This can be observed in the curvature of the photon paths and perhaps also in the curvature of the graviton paths when their existence becomes eventually proven by detection. 
Table 1. Transformation formulas for parameters from the region with a gravitational field

\begin{tabular}{|c|c|c|c|}
\hline Parameter name & $\begin{array}{l}\text { Parameter value } \\
\text { in the } g \text { field }\end{array}$ & $\begin{array}{l}\text { Stationary local } \\
\text { observer value }\end{array}$ & $\begin{array}{l}\text { Parameter value } \\
\text { out of the } g \text { field }\end{array}$ \\
\hline Radial speed of light & $c_{i}=c g_{t t}$ & $c_{l o}=c \sqrt{g_{t t}}$ & $c$ \\
\hline Dielectric constant & $\varepsilon_{i}=\varepsilon / g_{t t}$ & $\varepsilon_{l o}=\varepsilon / \sqrt{g_{t t}}$ & $\varepsilon$ \\
\hline Photon linear momentum & $p_{i}=p / \sqrt{g_{t t}}$ & $p_{l o}=p$ & $p=E / c$ \\
\hline Photon wavelength & $\lambda_{i}=\lambda \sqrt{g_{t t}}$ & $\lambda_{l o}=\lambda$ & $\lambda=c / v$ \\
\hline Photon energy & $E_{i}=E \sqrt{g_{t t}}$ & $E_{l o}=E \sqrt{g_{t t}}$ & $E=h v$ \\
\hline Falling inertial mass & $m_{i}=m / g_{t t}$ & $m_{l o}=m / \sqrt{g_{t t}}$ & $m$ \\
\hline Falling particle energy & $E_{i}=E g_{t t}$ & $E_{l o}=E \sqrt{g_{t t}}$ & $E=m c^{2}$ \\
\hline Frequency & $v_{i}=v \sqrt{g_{t t}}$ & $v_{l o}=v \sqrt{g_{t t}}$ & $v$ \\
\hline Time increment & $d t_{i}=d t / \sqrt{g_{t t}}$ & $d t_{l o}=d t / \sqrt{g_{t t}}$ & $d t$ \\
\hline Temperature & $T_{i}=T \sqrt{g_{t t}}$ & $T_{l o}=T \sqrt{g_{t t}}$ & $T$ \\
\hline * Particle linear momentum & $p_{i}=p$ & $p_{l o}=p$ & $p=m v$ \\
\hline * Boltzmann constant & $k_{B i}=k_{B}$ & $k_{B l o}=k_{B}$ & $k_{B}$ \\
\hline * Planck constant & $h_{i}=h$ & $h_{l o}=h$ & $h$ \\
\hline * Charge & $q_{i}=q$ & $q_{l o}=q$ & $q$ \\
\hline * Fine structure constant & $\alpha_{i}=\alpha$ & $\alpha_{l o}=\alpha$ & $\alpha=q^{2} / 2 \varepsilon h c$ \\
\hline
\end{tabular}

Note: The asterisk $(*)$ designates the parameters that are independent of the gravitational potential. 